ARTIGO ORIGINAL

\title{
Produção de energia da madeira de espécies da Caatinga aliada ao manejo florestal sustentável
}

\author{
Caatinga wood energy production coupled with sustainable forest \\ management
}

\author{
Ana Carolina de Carvalho ${ }^{1}$ (D), Rosimeire Cavalcante dos Santos ${ }^{1}$ (D), Renato Vinícius Oliveira Castro ${ }^{2}$ (i) \\ Cynthia Patricia de Sousa Santos ${ }^{1}$ (D), Sarah Esther de Lima Costa ${ }^{1}$ (D), \\ Adailton José Epaminondas de Carvalho ${ }^{3}$ (D), Frans Germain Corneel Pareyn ${ }^{4}$ (D), \\ Graziela Baptista Vidaurre ${ }^{5}$ (D), Ananias Francisco Dias Junior ${ }^{5}$ (D), Maria Naruna Felix de Almeida ${ }^{5}$ (D) \\ ${ }^{1}$ Universidade Federal do Rio Grande do Norte - UFRN, Macaíba, RN, Brasil \\ ¿Universidade Federal de São João del-Rei - UFSJ, São João del-Rei, MG, Brasil \\ ${ }^{3}$ Florescer Projetos e Consultoria - FPC, Natal, RN, Brasil \\ ${ }^{4}$ Associação Plantas do Nordeste - APNE, Recife, PE, Brasi \\ ${ }^{5}$ Universidade Federal do Espírito Santo - UFES, Jerônimo Monteiro, ES, Brasil
}

Como citar: Carvalho, A. C., Santos, R. C., Castro, R. V. O., Santos, C. P. S., Costa, S. E. L., Carvalho, A. J. E., Pareyn, F. G. C., Vidaurre, G. B., Dias Junior, A. F., \& Almeida, M. N. F. (2020). Produção de energia da madeira de espécies da Caatinga aliada ao manejo florestal sustentável. Scientia Forestalis, 48(126), e3086.

https://doi.org/10.18671/scifor.v48n126.08

\section{Resumo}

O uso sustentável da madeira de florestas nativas está condicionado a estudos que investiguem a produtividade aliada às características tecnológicas da madeira, pois possibilita entender a quantidade de energia disponível no povoamento. O objetivo deste estudo foi avaliar a produtividade da madeira e a produção de energia por unidade de produção anual (UPA), além de realizar a estimativa da idade de rotação do povoamento, em uma área manejada após corte raso. O estudo foi realizado no município de João Câmara/RN. Do total de 15 unidades de produção anual que compõem o plano de manejo da área foram selecionados quatro em diferentes idades de corte. De acordo com um delineamento inteiramente casualizado, foram avaliadas dez parcelas retangulares $(20 \mathrm{~m} \times 20 \mathrm{~m}$ ) por unidade de produção anual e, após realizada a análise do inventário das parcelas foram selecionadas as espécies com no mínimo 70\% de representatividade da área: Poincianella pyramidalis (Tul.) L. Q. Queiroz, Capparis flexuosa L, Ziziphus joazeiro Mart, Piptadenia stipulacea (Benth.) Ducke, Mimosa tenuiflora (Willd.) Poiret, Croton sonderianus Müll.Arg e Aspidosperma pyrifolium Mart. Foram abatidas três árvores-amostra de cada espécie, sendo posteriormente cubadas e, do seu tronco, retiradas amostras à altura de 1,30 m do solo (DAP) para determinação da densidade básica, composição imediata, poder calorífico superior da madeira, além da densidade energética e estoque de energia no povoamento. A UPA 1 apresentou maior produção em volume de madeira e as espécies Mimosa tenuiflora, Piptadenia stipulacea e Poincianella pyramidalis apresentaram as maiores densidades energéticas. Dessa forma, a UPA 1 se destacou por apresentar maior estoque de energia. A partir do ajuste das curvas de crescimento e de produção foi possível estimar que para as condições da área a idade de rotação ou de corte das UPA deve ser de 17,3 anos.

Palavras-chave: Biomassa energética; Semiárido brasileiro; Modelagem.

\section{Abstract}

The sustainable use of wood from native forests is conditioned to studies that investigate productivity combined with the technological characteristics of wood, since it makes it possible to understand the amount of energy available in the stands. The objective of this study was to evaluate wood productivity

Fonte de financiamento: Coordenação de Aperfeiçoamento de Pessoal de Nível Superior - Brasil (CAPES) - Código Financeiro 001.

Conflito de interesse: Nada a declarar.

Autor correspondente: engfcarolcarvalho@gmail.com

Recebido: 20 setembro 2018.

Aceito: 17 junho 2019.

Editor: Francides Gomes Silva Júnior

(i) Este é um artigo publicado em acesso aberto (Open Access) sob a licença Creative Commons Attribution, que permite uso, distribuição e reprodução em qualquer meio, sem restrições desde que o trabalho original seja corretamente citado. 
and energy production per unit of annual production, besides estimating the rotation age of the stands in an area managed after shallow cutting. The study was carried out in the municipality of João Câmara / RN. Of the total of 15 annual production units that make up the area management plan, four were selected at different cutting ages. According to a completely randomized design, ten rectangular plots (20 mx $20 \mathrm{~m}$ ) per annual production unit were evaluated and, after analyzing the plot inventory, the species with a minimum of $70 \%$ representative of the area were selected: Poincianella pyramidalis (Benth.) Ducke, Mimosa tenuiflora (Willd.) Poiret, Croton sonderianus Müll.Arg and Aspidosperma pyrifolium Mart. Three sample trees of each species were felled, and then samples were taken at a height of $1.30 \mathrm{~m}$ from the soil $(\mathrm{DBH})$ to determine the basic density, immediate composition, higher calorific value of the wood, and energy density and stock of energy in the stand. UPA 1 presented higher volume production of wood and the species Mimosa tenuiflora, Piptadenia stipulacea and Poincianella pyramidalis had the highest energy densities. In this way, UPA 1 stood out for having a higher energy stock. From the adjustment of the growth and production curves it was possible to estimate that for the conditions of the area the age of rotation or cut of the UPA should be 17.3 years.

Keywords: Energy biomass; Brazilian semi-arid; Modeling.

\section{INTRODUÇÃO}

A extração da madeira para utilização nos fins florestais pode tornar-se uma atividade insustentável quando não realizada numa área sob manejo florestal sustentável, principalmente para desígnio dos segmentos industriais que demandam elevada quantidade de madeira. Os impactos do desmatamento ilegal podem acarretar consequências negativas na exploração madeireira e na extração de produtos florestais não madeireiros (Carvalho, 2017).

Todavia, ao se observar o uso da madeira pelo setor industrial como uma das principais fontes de energia no estado do Rio Grande do Norte, a indústria de cerâmica vermelha se configura como uma das maiores consumidoras de madeira explorada e comercializada pelos proprietários de áreas sob manejo florestal no estado, com destaque para o consumo na região do Seridó, de clima semiárido, onde está localizado o principal polo cerâmico (Silva, 2008; Tavares, 2014; Schwob et al., 2017). Tal aspecto torna-se ainda mais relevante pelo fato do bioma que abrange o estado, a Caatinga, ser caracterizado pelo uso intensivo e inadequado das espécies florestais, condicionando a elevados índices de extinção (Silva \& Oren, 1997; Milliken et al., 2018).

A demanda por produtos energéticos nessa região é crescente, aumentando assim a pressão sobre a flora nativa para obter de forma prioritária a lenha para combustão direta (Paes et al., 2012; Dias Júnior et al., 2018). É muito importante conhecer o potencial energético de uma espécie a partir da quantidade de calor desprendida da madeira. A capacidade energética de determinada espécie é influenciada, principalmente, pela composição química da madeira, particularmente pelos teores de extrativos e lignina (Demirbas, 2001).

Apesar da importância da vegetação da região do semi-árido como fonte de energia, é grande a carência de informações sobre o material lenhoso obtido de manejo florestal sustentável para esta finalidade. Tal fato é reforçado pela extração ilegal da madeira da Caatinga, o qual não existe dados publicados sobre essa quantidade, e dessa forma dificulta estimar o consumo real de madeira no estado e realizar inferências sobre a produção e comercialização da madeira legalizada, aliada à demanda de lenha. O conhecimento desses dados poderia contribuir com práticas mais efetivas de recuperação de áreas exploradas, pelo manejo florestal sustentável de múltiplas espécies (Riegelhaupt et al., 2010; Amazonas et al., 2018).

Em se tratando da avaliação do potencial energético a partir do uso sustentável da madeira na região do Semiárido, conhecer a produtividade da madeira e das suas propriedades físicas e químicas atreladas às atividades de manejo é relevante; uma vez que tanto o conhecimento sobre a quantidade de energia estocada na madeira, quanto sobre o volume real no momento da exploração e na prospecção futura pode resultar na estabilização de metodologias com alcance de resultados socioeconômicos favoráveis (Trugilho et al., 2015; Brand, 2017; Dias Júnior et al., 2018). Em geral, a Caatinga apresenta estratos arbóreo-arbustivos e fustes tortuosos, o que inviabiliza o uso dos maciços florestais para outras finalidades a não ser para a energética. 
Na região do Semi-árido brasileiro, especificamente, no Estado do Rio Grande do Norte, não há registros de informações científicas do potencial energético sob a perspectiva da produtividade volumétrica e qualidade da madeira das espécies da Caatinga, que proporcionem entendimentos sobre o seu ciclo de corte adotado para as áreas sob manejo florestal. Tais atributos estariam ligados aos estudos que envolvam as áreas de manejo florestal, mensuração, exploração e regeneração do incremento anual das espécies, de modo a relacionar produtividade volumétrica à qualidade da madeira para uso na geração de energia térmica, considerando que esse combustível encontra-se presente em diversas atividades representativas no estado como as indústrias de cal, gesso, cimento e cerâmica vermelha (Riegelhaupt et al., 2010; Brand, 2017).

Diante disso, é notória a necessidade de estudos que abordem a disponibilidade de energia da madeira comercializada de forma legal, após exploração de corte raso em função da condução de manejo florestal sustentável, subsidiando informações que poderão auxiliar nas decisões quanto ao ciclo de corte das áreas manejadas objetivando a geração de energia por meio do uso da madeira como lenha. A hipótese levantada neste estudo é a de que há de se considerar a produção em lenho, após corte raso, aliada às características tecnológicas da madeira para obter respostas sobre o potencial de produção de energia da madeira em áreas sob manejo, pois dessa maneira será possível conjugar informações sobre estimativa da idade de rotação do povoamento.

Portanto, o objetivo deste estudo foi avaliar a produtividade volumétrica, as propriedades energéticas da madeira e a estimativa da idade de rotação do povoamento em uma área manejada, após corte raso.

\section{MATERIAL E MÉTODOS}

\section{Caracterização da área de estudo}

O estudo foi realizado na Fazenda Milhã/Poço da Pedra que possui 1.132,78 ha de mata nativa de Caatinga sob manejo florestal sustentável, representando aproximadamente $60 \%$ da área total da propriedade. A área está situada nas coordenadas $5^{\circ} 35^{\prime} 47.3^{\prime \prime} \mathrm{S} ; 35^{\circ} 51^{\prime \prime} 59.6^{\prime \prime} \mathrm{W}$, na região Agreste, microrregião de Serra Verde, entre os municípios de Jardim de Angicos e João Câmara, a 100 km de Natal, capital do Estado do Rio Grande do Norte (Figura 1).

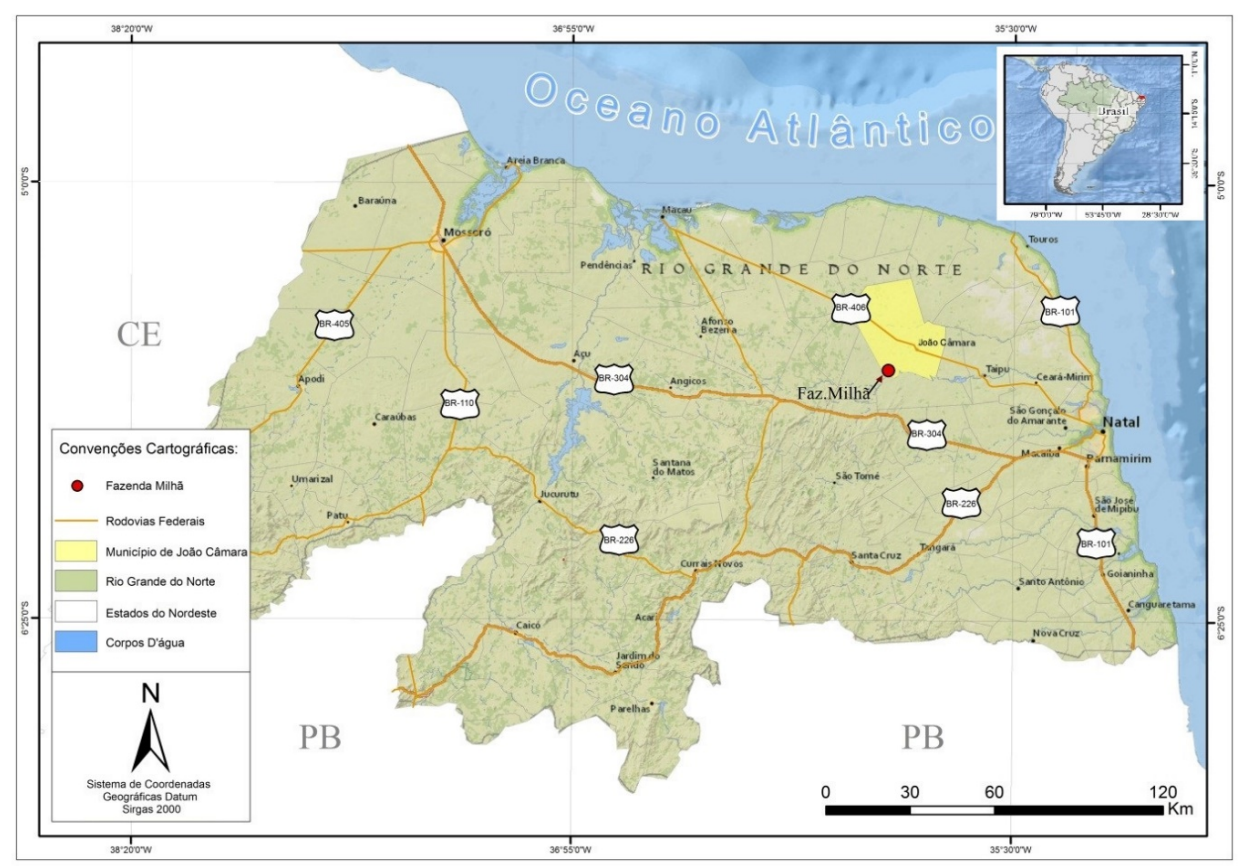

Figura 1. Mapa do Estado do Rio Grande do Norte, com destaque para o município de João Câmara/RN, Brasil, onde está localizada a área de estudo. 
A área encontra-se sob manejo florestal sustentável para exploração de madeira visando uso energético, desde o ano de 2003. No entanto, os cortes ocorreram de acordo com a demanda e 10 unidades de produção anual foram exploradas (corte raso) (Instituto de Desenvolvimento Sustentável e Meio Ambiente do Estado do Rio Grande do Norte, 2009). O ciclo de corte adotado na Fazenda Milhã/Poço da Pedra foi de 15 anos, respeitando o tempo mínimo proposto para a rotação da Caatinga (Brasil, 2009). De acordo com o mapeamento da cobertura florestal da área, realizado pelo Projeto Programa das Nações Unidas para o Desenvolvimento (PNUD)/Organização das Nações Unidas para Alimentação e Agricultura (FAO)/ Instituto Brasileiro do Meio Ambiente e dos Recursos Naturais Renováveis (IBAMA) (1992), a área pode ser classificada como Caatinga arbustiva arbórea fechada (tipo 3), apresenta um porte médio de árvores de 3 a $5 \mathrm{~m}$ de altura, com alto grau de cobertura dos solos com presença de sub-bosque.

O clima predominante é, segundo a classificação de Köppen, tropical chuvoso com verão seco e a estação chuvosa que se adianta para outono. A temperatura média anual encontra-se em torno de $26{ }^{\circ} \mathrm{C}$, com amplitude de $7^{\circ} \mathrm{C}$ e umidade relativa do ar com oscilação entre 50 e $80 \%$. Na área de estudo, há registros de precipitação média em torno de $900 \mathrm{~mm}$ anuais, com período chuvoso variando de fevereiro a setembro, irregularmente distribuída (Zakia et al., 1992). E com base nesses critérios, o Ministério da Integração Nacional, classificou a região onde está localizada a fazenda como semi-árido (Brasil, 2005a).

O solo é uma associação dos tipos podzólico vermelho-amarelo, equivalente eutrófico abrúptico plinthico, textura média fase caatinga hiperxerófila, relevo plano e planosol solódico a fraco, fase Caatinga hiperxerófila, relevo suave ondulado e solonetz solodizado, textura indiscriminada fase caatinga hiperxerófila relevo plano e suave ondulado (Carvalho, 2003).

\section{Distribuições das unidades amostrais}

Dentre os 15 unidades de produção anual que estão sob manejo florestal naquela área, foram selecionados quatro em função das idades de implantação (Figura 2), a saber: Unidade de produção anual 1 (T1): 12,3 anos; Unidade de produção anual 8 (T8): 9,3 anos; Unidade de produção anual 11 (T11): 7,2 anos e Unidade de produção anual 6 (T6): 6,6 anos da última exploração ou corte.

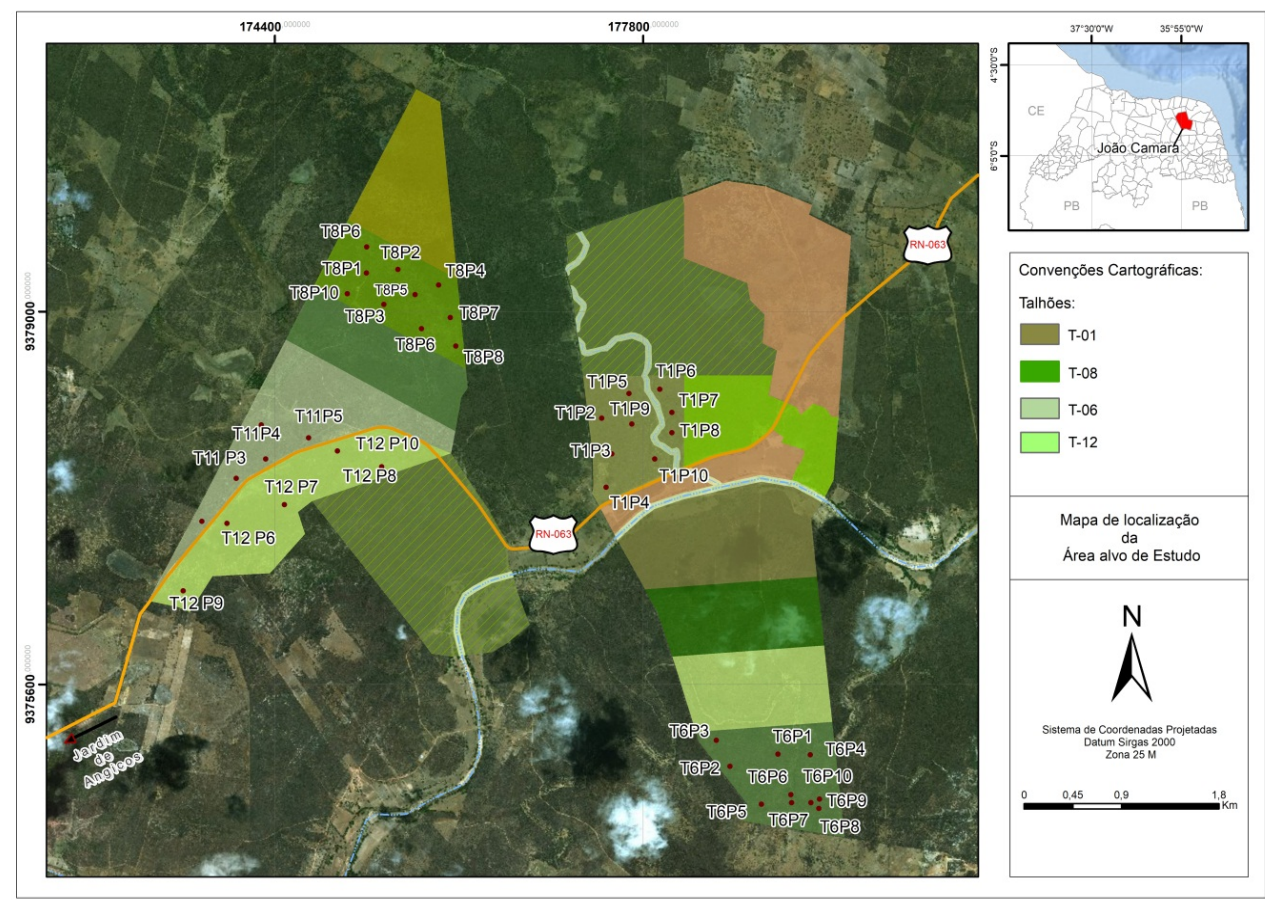

Figura 2. Unidades amostrais lançadas nos talhões em estudo. 
A partir do acesso ao plano de manejo da área foram distribuídas dez parcelas aleatórias em cada unidade de produção anual, totalizando quarenta pontos (Instituto de Desenvolvimento Sustentável e Meio Ambiente do Estado do Rio Grande do Norte, 2009). As parcelas foram previamente demarcadas, georreferenciadas e mapeadas com auxílio de um GPS Garmin 62s. Após a marcação do ponto localizado em campo, os quatro vértices foram demarcados com piquetes baixos $(0,50 \mathrm{~m})$ de madeira. Cada unidade amostral (parcela) foi dimensionada em $400 \mathrm{~m}^{2}(20 \mathrm{~m} \times 20 \mathrm{~m})$, considerando como aceitável um erro de $\pm 20 \%$ para o volume, com $95 \%$ de probabilidade (Brasil, 2005b).

$\mathrm{O}$ critério de inclusão das árvores a serem avaliadas foi o CAP (circunferência a altura o peito) $\geq 6,0 \mathrm{~cm}$, sendo mensurados com fita métrica a $1,30 \mathrm{~m}$ de altura. $O$ comprimento entre a base e o ápice de cada árvore (altura total) foi mensurado holisticamente, com aproximação de $10 \mathrm{~cm}$ (Rede de Manejo Florestal da Caatinga, 2005). A identificação das espécies foi por meio do nome vulgar e posterior confirmação do nome científico com auxílio de exsicata.

\section{Seleções das espécies representativas e determinação do volume em lenho}

A partir dos dados do inventário, selecionaram-se as espécies mais importantes por unidade de produção anual a partir do índice de valor de importância (IVI) da estrutura horizontal, que envolve os valores relativos médios entre a densidade (número de indivíduos por espécie), dominância (área basal da espécie) e frequência (proporção de parcela que aparece a espécie), segundo a Equação 1.

$I V I=\frac{[D R+D o R+F r]}{3}$

em que: IVI = Índice de Valor de Importância; $\mathrm{DR}=$ Densidade Relativa; DoR = Dominância Relativa; $\mathrm{Fr}=$ Frequência Relativa.

As espécies de maior IVI (com a soma acumulada maior ou igual a $70 \%$ do IVI total) foram derrubadas e posteriormente cubadas e retiradas amostras de madeira. Três árvores foram selecionadas por espécie de acordo com o diâmetro a altura do peito (DAP): uma árvore pequena $(\bar{x}-\sigma)$, uma média $(\bar{x})$ e uma grande $(\bar{x}+\sigma)$ de cada espécie. Dessa forma, as 21 árvores selecionadas, foram seccionadas a 0,30 m do solo e mensurados os diâmetros de seções ao longo do fuste de cada árvore, na base, à $0,50 \mathrm{~m}$, à 1,30 $\mathrm{m}$ e depois, a cada $0,50 \mathrm{~m}$ até a altura comercial da árvore (diâmetro de 1,6 cm).

A cubagem foi realizada pelo método de Smalian para a totalização do volume total com casca de cada seção. O volume total da árvore foi determinado pelo somatório dos volumes individuais de cada seção $\left(\mathrm{em}^{3}\right.$ ). $\mathrm{O}$ fator de forma foi calculado pela razão entre o volume real (obtido pela cubagem da árvore) e o volume dessa árvore considerando a sua forma igual a de um cilindro, e pela média dos fatores das árvores amostradas, ponderadas pelos respectivos IVI das espécies (Campos \& Leite, 2013; Husch et al., 2002):

$f f_{1,3}=\left(\frac{\text { Vreal }}{\text { Vcil }}\right)$

em que: $f f=$ Fator de forma; Vreal $=$ Volume real da árvore $\mathrm{em}^{3}$, determinado pela cubagem; Vcil = Volume do cilindro em $\mathrm{m}^{3}$, considerando a área seccional da árvore à 1,30 $\mathrm{m}$ $\left(\mathrm{em} \mathrm{m}^{2}\right)$ multiplicado pela altura total (em $\left.\mathrm{m}\right)$.

\section{Estimativas da idade de rotação no povoamento}

A idade de rotação foi determinada com base na curva de incremento ajustada utilizando as produtividades dos quatro unidades de produção anual de diferentes idades de regeneração após corte raso. Para isso a prognose e a idade de estagnação da área foi estudada. Essa estagnação definiu a idade de rotação (ciclo de corte). A equação que melhor 
representou os dados foi à função não linear e sigmoidal de Weibull. Para o ajuste dessa equação foi utilizado o software CurvExpert 1.3.

Tal função possui plasticidade, o que possibilita delinear diferentes tendências de crescimento (Campos \& Leite, 2013), apresentada na Equação 3.

$\operatorname{Volume}\left(\frac{m^{3}}{h a}\right)=\beta_{0}-\beta_{1} \cdot e^{-\beta_{2} \cdot \text { Idade }}{ }^{\beta_{3}}$

Para avaliar a idade ótima de corte foram calculados os incrementos correntes anuais (ICA) e os incrementos médios anuais (IMA) avaliam o quanto a floresta cresceu em volume no último ano e o crescimento médio da floresta até aquela idade, respectivamente (Imaña-Encinas et al., 2005). A idade de corte é definida pelo ponto máximo do IMA, que também coincide com o encontro das curvas de ICA e IMA (Campos \& Leite, 2013).

\section{Amostragem e análises das propriedades energéticas da madeira}

Com base nas estruturas horizontais das unidades de produção anual e considerando toda a área amostrada, definiu-se as sete espécies mais representativas e que refletem juntas, 90\% do IVI (Tabela 1), para seleção de árvores-amostra. Foram retirados discos de madeira com $15 \mathrm{~cm}$ de comprimento e espessuras variadas Em virtude do pequeno diâmetro das espécies da Caatinga localizados no DAP de cada uma das 21 árvores definidas previamente de acordo com o levantamento feito na área de estudo, de modo que o abate de tais árvores foi realizado nos unidades de produção anual de forma aleatória.

Tabela 1. Espécies selecionadas para a cubagem e amostragem da madeira.

\begin{tabular}{cc}
\hline Nome Científico & Nome vulgar \\
\hline Poincianella pyramidalis (Tul.) L. Q. Queiroz & Catingueira \\
Capparis flexuosa L & Feijão bravo \\
Ziziphus joazeiro Mart & Juazeiro \\
Piptadenia stipulacea (Benth.) Ducke & Jurema branca \\
Mimosa tenuiflora (Willd.) Poiret & Jurema preta \\
Croton sonderianus Müll.Arg & Marmeleiro \\
Aspidosperma pyrifolium Mart & Pereiro
\end{tabular}

Em seguida, diante da composição das unidades de produção anual anteriormente apresentado, os materiais lenhosos relativos a cada uma das espécies, tiveram uma parte dos discos cavaqueados e em seguidas triturados em um moinho Willey para a obtenção de um material mais fino e homogêneo, com granulometria entre as peneiras de 40 e 60 mesh.

A outra parte que não foi processada (as cunhas) foi utilizada para a determinação da densidade básica, determinada pelo método de imersão em água, após completa saturação das amostras de madeira expressando seu valor como a média de duas cunhas opostas por disco NBR 11941 (Associação Brasileira de Normas Técnicas, 2003).

Para a determinação da composição imediata, os procedimentos seguiram as descrições da ASTM E872-1982 (American Society for Testing and Materials, 2006) e ASTM E1755-2001 (American Society for Testing and Materials, 2007), para determinação dos teores de materiais voláteis, teor de carbono fixo (por diferença) e teor de cinzas.

O poder calorífico superior foi determinado em uma bomba calorimétrica adiabática, IKA C200, conforme metodologia descrita na norma ASTM D2015-1996 (American Society for Testing and Materials, 2000). Com base nesses parâmetros a densidade energética foi calculada pelos produtos da densidade básica multiplicado pelo poder calorífico da madeira de cada uma das espécies analisadas. 
em que: $\mathrm{DE}=$ Densidade energética $\left(\mathrm{kcal} / \mathrm{m}^{3}\right) ; \mathrm{PCS}=$ Poder calorífico superior $(\mathrm{kcal} / \mathrm{kg})$; $\mathrm{DB}=$ Densidade básica $\left(\mathrm{kg} / \mathrm{m}^{3}\right)$.

\section{Análise dos dados}

Os dados foram analisados por meio de um delineamento inteiramente casualizados (DIC), com três repetições. As propriedades de cada espécie foram submetidas ao teste F da análise de variância e quando confirmados os efeitos das espécies nas propriedades, estas foram comparadas pelo teste de médias Tukey, sempre à 95\% de probabilidade.

\section{RESULTADOS E DISCUSSÃO}

O levantamento florestal permitiu a obtenção da análise fitossociológica e a identificação de 24 espécies arbóreas nas quatro unidades de produção anual estudadas, onde se observou também a sucessão das espécies. Essa sucessão de espécies é uma tendência e, ocorre porque as populações destinam-se a modificar o ambiente em que estão gerando condições favoráveis para outras populações (Odum, 2001).

O fator de forma médio das árvores amostradas foi de 0,91. Valor esse, correlato aos encontrados em outros estudos para a mesma vegetação por Souza et al. (2016) e Zakia et al. (1992) com 0,88 e 0,90, respectivamente. Da mesma forma, análogo ao recomendado pela Rede de Manejo Florestal da Caatinga (2005) que é de 0,90. Esse fator foi utilizado para calcular o volume de todas as árvores de todas as parcelas e unidades de produção anual.

É sabido que quanto mais velha a unidade de produção anual (corte mais antigo), maior será a sua regeneração em lenho e, consequentemente, maior produtividade volumétrica (Tabela 2). Esse aumento na produtividade de madeira observado no estudo é consequência da sucessão ecológica no povoamento florestal, pois o volume em madeira tende a aumentar, devido ao crescimento das árvores durante a regeneração. No entanto, é importante considerar que cada espécie possui uma estocagem energética diferente, ou seja, a variação da constituição química, densidade básica e poder calorífico da madeira permitirá a diferença na densidade energética entre as espécies.

Tabela 2. Volume médio por talhão com diferentes idades.

\begin{tabular}{cc}
\hline Talhão & Volume $\mathbf{~ m e ́ d i o ~}\left(\mathbf{m}^{\mathbf{3}} \mathbf{h} \mathbf{a}^{-\mathbf{1}}\right)$ \\
\hline $1(12,3$ anos $)$ & 29,05 \\
$8(9,3$ anos $)$ & 3,95 \\
$12(7,2$ anos $)$ & 1,24 \\
$6(6,6$ anos $)$ & 1,04 \\
$C V(\%)=43,4 \%$ e $\mathrm{E} \%=13,2$ & \\
\hline
\end{tabular}

Com base no inventário dos quatro unidades de produção anual e da utilização de estimativas de produção, foi possível ajustar curvas de crescimento visando estimar a produção futura em lenho. A equação de Weibull foi a função que melhor descreveu a curva de incremento (Figura 3). 

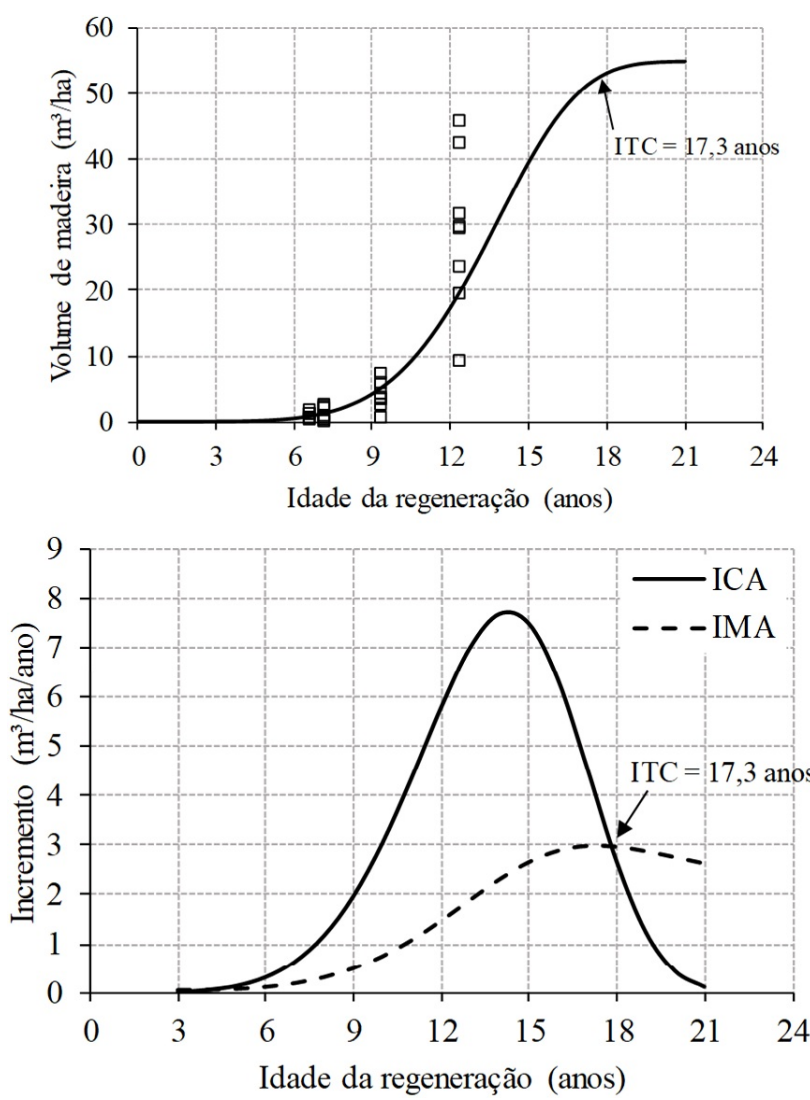

Figura 3. Incrementos volumétricos médios e corrente anuais (IMA e IMC) considerando a idade de regeneração e Idade técnica de corte (ITC).

Considerando que a curva de Incremento corrente anual (ICA) refere-se ao volume de madeira acumulado no último ano e que o Incremento médio anual (IMA) corresponde à média de volume acumulado até a idade de corte, o ICA atinge o máximo antes da curva do IMA (Rodriguez, 1991). A idade de máximo IMA pode ser facilmente identificada pelo cruzamento das curvas de IMA e ICA (Figura 3). As linhas mostram a evolução de IMA e ICA, tornando possível avaliar o ponto de maturação para corte quando do cruzamento dessas duas curvas. Assim, tem-se definida a idade de corte segundo o critério volumétrico quando as duas curvas tracejada e cheia se cruzam no ponto de máximo IMA.

Apesar da idade de corte indicada em planos de manejo florestal sustentável na caatinga ser de 15 anos (Meunier, 2014), a curva de incremento apresentada na Figura 3, demonstra que, para os dados avaliados, a idade técnica de corte (ITC) é de 17,3 anos, visto que esse tempo é o mais indicado quando se observa a regeneração da vegetação do local.

As maiores densidades da madeira foram observadas para as espécies Mimosa tenuiflora e Piptadenia stipulacea, e dentre as 7 espécies de maior IVI, Mimosa tenuiflora, como pode-se observar na Figura 4. A densidade básica da madeira das demais espécies variaram de $587 \mathrm{~kg} / \mathrm{m}^{3}$ a $758 \mathrm{~kg} / \mathrm{m}^{3}$, sendo a Mimosa tenuiflora $\left(758 \mathrm{~kg} / \mathrm{m}^{3}\right)$ e a Poincianella pyramidalis $\left(736 \mathrm{~kg} / \mathrm{m}^{3}\right)$ as espécies com as maiores densidades básicas da madeira. Ao se considerar a densidade de madeira de espécies comumente utilizadas para uso na geração de energia (como as de eucalipto), esses valores são tecnicamente viáveis. 


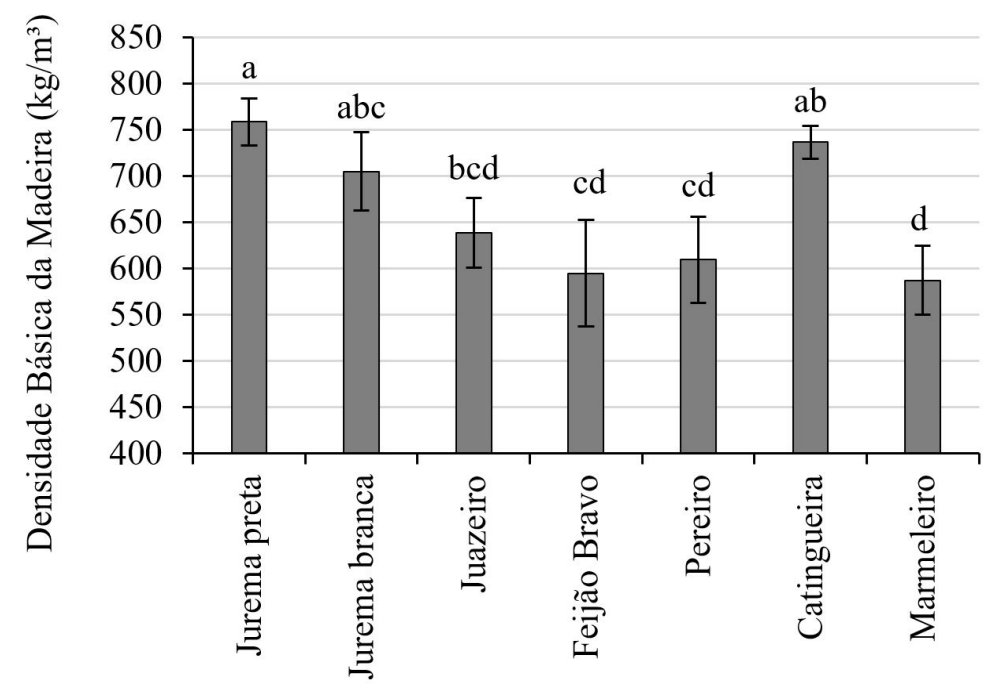

Figura 4. Densidade básica da madeira das espécies estudadas. Médias seguidas da mesma letra entre espécies, para uma mesma variável, não diferem entre si, a 5\% de significância, pelo teste Tukey.

A velocidade da queima bem como a produção de energia está diretamente relacionada à densidade, onde o uso de madeiras com baixa densidade implica em uma queima rápida e numa menor produção de energia por unidade de volume (Demirbas, 2003). No entanto, quando a densidade é muito elevada ocorre dificuldade de um bom desempenho da combustão, principalmente, em função das dimensões do material combustível (Dias Júnior et al., 2015). Todavia, o estoque de energia é maior em madeiras com maiores densidades em função de maior massa disponível para a geração de energia.

Observa-se que o menor teor de materiais voláteis da madeira $(77,03 \%)$ foi detectado na madeira de Mimosa tenuiflora, e o maior teor (82,45\%), detectado na madeira da espécie Piptadenia stipulacea, sendo estes diferentes estatisticamente. No entanto, as demais espécies não apresentaram diferenças significativas, compreendendo o intervalo entre as duas espécies anteriormente supracitadas (Figura 5). O alto teor de voláteis torna a reatividade do combustível maior, pois a partir da volatilização, os gases reagem com o oxigênio presente no ar e promovem a combustão (Pereira et al., 2000; Klautau, 2008).

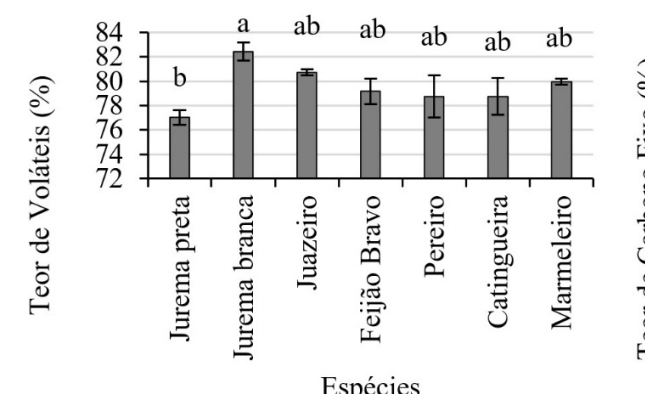

Espécies

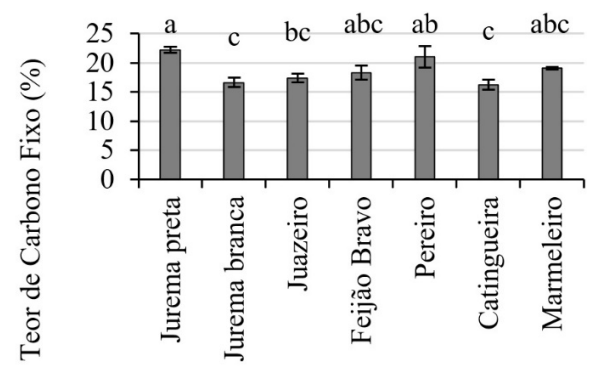

Espécies

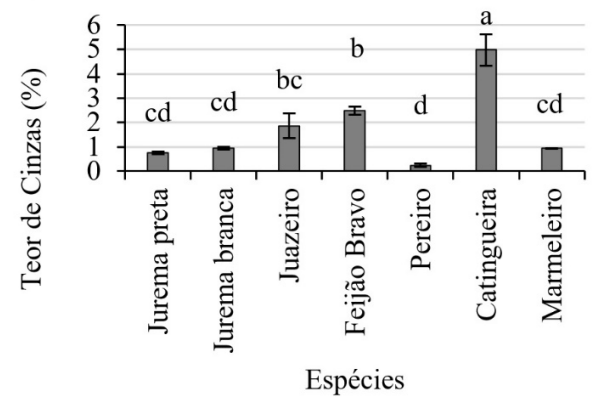

Figura 5. Composição imediata das espécies estudadas. Médias seguidas da mesma letra entre as espécies, para uma mesma variável, não diferem entre si, a 5\% de significância, pelo teste Tukey. 
As espécies Mimosa tenuiflora, Aspidosperma pyrifolium e Croton sonderianus foram as que apresentaram maiores valores médios para o teor de carbono fixo na madeira, 22,22\%, $21,02 \%$ e $19,08 \%$, respectivamente, os quais não diferiram entre si. Já a Piptadenia stipulacea e a Poincianella pyramidalis apresentaram os menores valores, $16,61 \%$ e $16,25 \%$, respectivamente, e também não apresentaram diferença significativa entre si. A variação do carbono fixo da encontra-se na faixa entre $15 \%$ e $25 \%$ o que é esperado para madeiras de folhosas (Brito \& Barrichelo, 1982). A variação dos teores de cinzas da madeira foi de 0,23\% para a Aspidosperma pyrifolium e 4,98\% para a Poincianella pyramidalis.

De forma geral, os valores para os teores de materiais voláteis e de carbono fixo se encontram de acordo com o apresentado pela literatura para a maioria das madeiras de espécies lenhosas, sendo o teor de carbono fixo a variável mais desejável para uso em combustão direta de biomassa (Demirbas, 2001; Kumar et al., 2010). No entanto, elevados teores de cinzas na madeira não é desejável, pois, possivelmente, irá resultar em um elevado conteúdo de minerais nas caldeiras de queima, podendo ainda comprometer o poder calorífico do combustível (Dias Júnior et al., 2018).

O poder calorífico superior da madeira das sete espécies variou de $4.897 \mathrm{kcal} / \mathrm{kg}$ para Mimosa tenuiflora a $4.011 \mathrm{kcal} / \mathrm{kg}$ para o Ziziphus joazeiro. As espécies que apresentaram os menores valores de poder calorífico foram Piptadenia stipulacea, Mimosa tenuiflora, Croton sonderianus e Aspidosperma pyrifolium (Figura 6) também apresentam, de modo geral, os maiores valores médios de teores de cinzas (Figura 5).
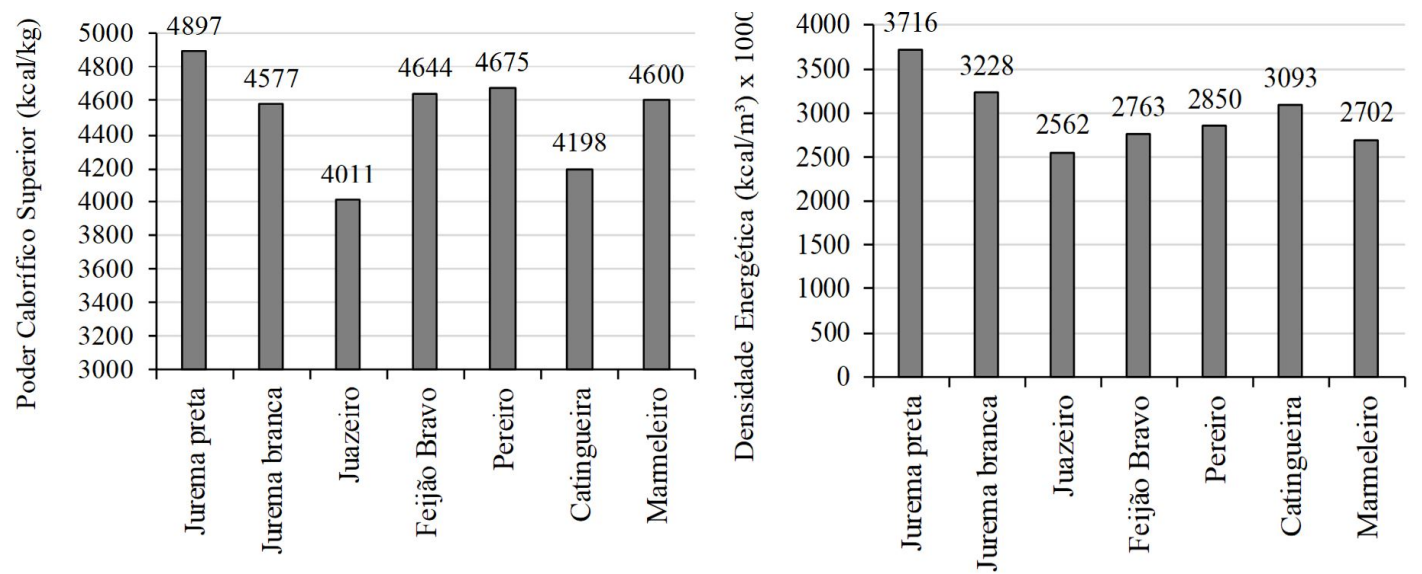

Figura 6. Poder calorífico superior e densidade da madeira das sete espécies estudadas.

A elevação do teor de carbono fixo incrementa o poder calorífico, uma vez que a madeira libera energia por mais tempo durante o processo de combustão (Soares et al., 2015; Silva et al., 2014). Segundo os mesmos autores, o carbono fixo existente na madeira é a principal fonte de energia para a combustão, e consequentemente, a correlação entre o poder calorífico e os materiais voláteis e entre os materiais voláteis e as cinzas, são negativas. Santos et al. (2013) obtiveram valores de $4.442 \mathrm{kcal} \mathrm{kg}^{-1}$ para a madeira de catingueira (Poincianella pyramidalis), resultado semelhante ao encontrado neste trabalho.

Ao se considerar a quantidade de energia por unidade de volume, pode-se destacar a espécie Mimosa tenuiflora que apresentou densidade energética de $3.716 \mathrm{kcal} \mathrm{m}^{-3}$ (Figura 6) a alta densidade energética está relacionada ao elevado poder calorífico superior e densidade básica da madeira.

A densidade energética é proporcional à densidade básica da madeira, pois representa a quantidade de energia que pode ser liberada após a combustão completa de um determinado combustível (Protásio et al., 2012, 2013). Os diferentes valores médios de densidade energética podem ser atribuídas às distintas densidades da madeira. A densidade básica pode ser considerada o principal índice de qualidade para o uso energético dos combustíveis de biomassa, pois influencia diretamente a densidade energética.

Analisando a Figura 7, conclui-se que ao relacionar a energia estocada ( $\mathrm{kcal} \mathrm{ha}^{-1}$ ) com a idade dos diferentes talhões, o com maior tempo de implantação $(12,3$ anos) se sobressai 
aos demais. Através de tal informação vê-se a importância da prática do manejo florestal, visto que com o mesmo pode-se obter o aumento na produtividade de madeira.

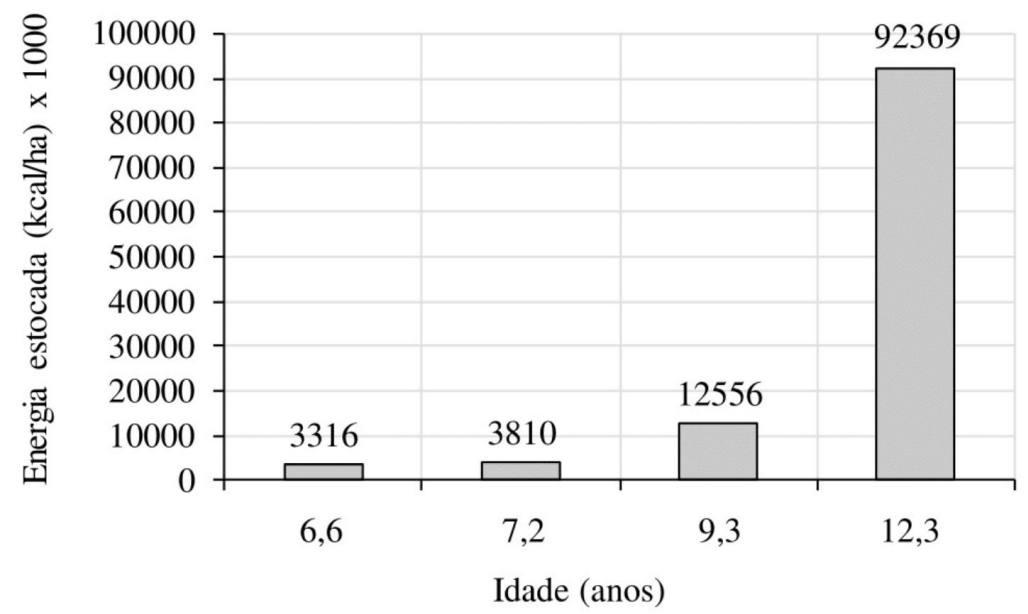

Figura 7. Energia estocada em kcal/ha por idade dos talhões em anos.

A quantidade de energia estocada é superior quando mais velha é a unidade de produção anual (maior intervalo de corte). Isso se deve em grande parte a estrutura da unidade de produção anual, visto que por ser mais antigo apresenta uma maior diversidade de espécie, além de possuir indivíduos mais robustos. Essa característica tende a aumentar conforme se aumenta esse intervalo de corte da área, de modo a corroborar com a importância de manejar as áreas de interesse para extração de lenha.

\section{CONCLUSÕES}

Conclui-se que houve maior produtividade volumétrica em lenho na UPA 1 e por consequência das características energéticas da madeira, bem como a produção em volume de madeira, o maior estoque de energia também é observado na UPA 1 (12,3 anos). Vale salientar que a Mimosa tenuiflora, Piptadenia stipulacea e Poincianella pyramidalis foram as espécies que apresentaram maiores densidades energéticas.

O cruzamento das curvas de incremento e de produção (ICA e IMA) para estimativas da produção futura sugere o corte das espécies da Caatinga em idade (de rotação) de 17,3 anos para regeneração diferentemente da idade de rotação utilizada atualmente que é de 15 anos.

\section{REFERÊNCIAS}

Amazonas, N. T., Forrester, D. I., Silva, C. C., Almeida, D. R. A., Rodrigues, R. R., \& Brancalion, P. H. S. (2018). High diversity mixed plantations of Eucalyptus and native trees: an interface between production and restoration for the tropics. Forest Ecology and Management, 417, 247-256. http://dx.doi.org/10.1016/j.foreco.2018.03.015.

American Society for Testing and Materials - ASTM. (2000). ASTM D2015-1996: standard test method for gross calorific value of coal and coke by the adiabatic bomb calorimeter. West Conshohocken: ASTM International.

American Society for Testing and Materials - ASTM. (2006). ASTM E872-1982: standard test method for volatile matter in the analysis of particulate wood fuels. West Conshohocken: ASTM International.

American Society for Testing and Materials - ASTM. (2007). ASTM E1755-2001: standard test method for ash in biomass. West Conshohocken: ASTM International.

Associação Brasileira de Normas Técnicas - ABNT. (2003). ABNT NBR 11491: madeira: determinação da densidade básica. Rio de Janeiro: ABNT.

Brand, M. A. (2017). Potencial de uso da biomassa florestal da caatinga, sob manejo florestal sustentável, para geração de energia. Ciência Florestal, 27(1), 117-127. http://dx.doi.org/10.5902/1980509826452. 
Brasil. Ministério da Integração Nacional - MIN. Secretária de Desenvolvimento Regional. (2005a). Nova delimitação do Semi-árido brasileiro. Brasília: MIN.

Brasil. Ministério do Meio Ambiente. (2005b). Análise das variações da biodiversidade do bioma Caatinga: suporte a estratégias regionais de conservação (Biodiversidade, No. 12). Brasília: MMA.

Brasil. Ministério do Meio Ambiente. (2009, 26 de junho). Dispõe sobre procedimentos técnicos para elaboração, apresentação, execução e avaliação técnica de Planos de Manejo Florestal Sustentável PMFS da Caatinga e suas formações sucessoras, e dá outras providências (Instrução normativa ${ }^{\circ}$ 1, de 25 de junho de 2009). Diário Oficial [da] República Federativa do Brasil, Brasília.

Brito, J. O., \& Barrichelo, L. E. G. (1982). Aspectos técnicos da utilização da madeira e carvão vegetal como combustíveis. In Anais do $2^{\circ}$ Seminário de Abastecimento Energético Industrial com Recursos Florestais (pp. 101-137). São Paulo; Governo do Estado de São Paulo.

Campos, J. C. C., \& Leite, H. G. (2013). Mensuração florestal: perguntas e respostas (4. ed.). Viçosa: Editora UFV.

Carvalho, A. J. E. (2003). Plano de manejo florestal: Fazenda Milhã/Poço da Pedra. Natal: Florescer Projetos e Consultoria Ambiental Ltda.

Carvalho, A. L. (2017). Regeneração natural de uma área manejada na Floresta Estadual do Antimary, estado do Acre (Tese de doutorado). Programa de Pós-graduação em Ciências de Florestas Tropicais, Instituto Nacional de Pesquisas da Amazônia, Manaus.

Demirbas, A. (2001). Biomass resource facilities biomass conversion processing for fuels and chemicals. Energy Conversion and Management, 42(11), 1357-1378. http://dx.doi.org/10.1016/S01968904(00)00137-0.

Demirbas, A. (2003). Relationships between lignin contents and fixed carbon contents of biomass samples. Energy Conversion and Management, 44(9), 1481-1486. http://dx.doi.org/10.1016/S01968904(02)00168-1.

Dias Júnior, A. F., Andrade, C. R., Protásio, T. P., Melo, I. C. N. A., Brito, J. O., \& Trugilho, P. F. (2018). Pyrolysis and wood by-products of species from the Brazilian semi-arid region. Scientia Forestalis, 46(117), 65-75. http://dx.doi.org/10.18671/scifor.v46n117.06.

Dias Júnior, A. F., Brito, J. O., \& Andrade, C. R. (2015). Granulometric influence on the combustion of charcoal for barbecue. Revista Árvore, 39(6), 1127-1133. http://dx.doi.org/10.1590/010067622015000600016.

Husch, B., Beers, T. W., \& Kershaw Junior, J. A. (2002). Forest mensuration. New York: John Wiley \& Sons.

Imaña-Encinas, J., Silva, G. F., \& Pinto, J. R. R. (2005). Idade e crescimento das árvores (Comunicações Técnicas Florestais, Vol. 7, No. 1). Brasília: Departamento de Engenharia Florestal, Universidade de Brasília.

Instituto de Desenvolvimento Sustentável e Meio Ambiente do Estado do Rio Grande do Norte - IDEMA. (2009). Termo de referência para elaboração do plano de manejo florestal sustentável. Natal: IDEMA. Recuperado em 1 de setembro de 2018, de http://adcon.rn.gov.br/ACERVO/idema/DOC/DOC000000000005854.PDF

Klautau, P. (2008). Análise experimental de uma fornalha a lenha de fluxo corrente para secagem de grãos (Dissertação mestrado). Universidade Federal do Paraná, Curitiba.

Kumar, R., Pandey, K. K., Chandrashekar, N., \& Mohan, S. (2010). Effect of tree-age on calorific value and other fuel properties of Eucalyptus hybrid. Journal of Forestry Research, 21(4), 514-516. http://dx.doi.org/10.1007/s11676-010-0108-x.

Meunier, I. M. J. (2014). Análises de sustentabilidade de planos de manejo florestal em Pernambuco (Tese de doutorado). Universidade Federal Rural de Pernambuco, Recife.

Milliken, W., Gasson, P., Pareyn, F., Sampaio, E. V. S. B., Lee, M., Baracat, A., Lima Araújo, E., \& Cutler, D. (2018). Impact of management regime and frequency on the survival and productivity of four native tree species used for fuelwood and charcoal in the caatinga of northeast Brazil. Biomass and Bioenergy, 116, 18-25. http://dx.doi.org/10.1016/j.biombioe.2018.05.010.

Odum, E. P. (2001). Ecologia. Rio de Janeiro: Guanabara Koogan.

Paes, J. B., Lima, C. R., Oliveira, E., \& Santos, H. C. M. (2012). Rendimento e caracterização do carvão vegetal de três espécies de ocorrência no semiárido brasileiro. Ciência da Madeira, 3(1), 1-10. http://dx.doi.org/10.12953/2177-6830.v03n01a01.

Pereira, J. C. D., Sturion, J. A., Higa, A. R., Higa, R. C. V., \& Shimizu, J. Y. (2000). Características da madeira de algumas espécies de eucalipto plantadas no Brasil (Documentos, No. 38). Colombo: Embrapa Florestas. 
Protásio, T. P., Couto, A. M., Reis, A. A., Trugilho, P. F., \& Godinho, T. P. (2013). Potencial siderúrgico e energético do carvão vegetal de clones de Eucalyptus spp. aos 42 meses de idade. Pesquisa Florestal Brasileira, 33(74), 137-149. http://dx.doi.org/10.4336/2013.pfb.33.74.448.

Protásio, T. P., Trugilho, P. F., Neves, T. A., \& Vieira, C. M. M. (2012). Análise de correlação canônica entre características da madeira e do carvão vegetal de Eucalyptus. Scientia Forestalis, 40(95), 317-326.

Rede de Manejo Florestal da Caatinga - RMFC. (2005). Protocolo de medições de parcelas permanentes. Recife: Comitê Técnico-Científico.

Riegelhaupt, E. M., Pareyn, F. G. C., \& Gariglio, M. A. (2010). O manejo florestal como ferramenta para o uso sustentável e conservação da Caatinga. In M. A. Gariglio, E. V. S. B. Sampaio, L. A. Cestaro \& P. Y. Kageyama (Eds.), Uso sustentável e conservação dos recursos florestais da Caatinga (pp. 349-367). Brasília: Serviço Florestal Brasileiro.

Rodriguez, L. C. E. (1991). Tópicos de Economia Florestal (Documentos Florestais, No. 12, pp. 1-49). Piracicaba: IPEF.

Santos, R. C., Carneiro, A. C. O., Pimenta, A. S., Castro, R. V. O., Marinho, I. V., Trugilho, P. F., Alves, I. C. N., \& Castro, A. F. N. M. (2013). Potencial energético da madeira de espécies oriundas de plano de manejo florestal no Estado do Rio Grande do Norte. Ciência Florestal, 23(2), 491-502. http://dx.doi.org/10.5902/198050989293.

Schwob, M. R. V., Henriques Junior, M. F., Carlos, M. E. M., Pacheco, L. F. L., \& Candido, R. S. (2017). Panorama do setor de cerâmica vermelha no Brasil. In M. F. Henriques Junior, J. A. P. Rodrigues (Eds.), Cerâmica Vermelha: Projeto EELA no Brasil (pp. 11-33). Rio de Janeiro: INT/MCTIC.

Silva, E. F. (Ed.). (2008). Diagnóstico do uso da lenha nas atividades agroindustriais do território do Seridó/RN. Caicó: Agência de Desenvolvimento Sustentável do Seridó.

Silva, J. M. C., \& Oren, D. C. (1997). Geographic variation and conservation of the Moustached Woodcreeper (Xiphocolaptes falcirostris), na endemic and threatened species of northeastern Brazil. Bird Conservation International, 7(3), 263-274. http://dx.doi.org/10.1017/S0959270900001568.

Silva, L. F., Paes, J. B., Jesus Junior, W. C., Oliveira, J. T. S., Furtado, E. L., \& Alves, F. R. (2014). Deterioração da madeira de Eucalyptus spp. por fungos xilófagos. Cerne, 20(3), 393-400. http://dx.doi.org/10.1590/01047760201420031462.

Soares, V. C., Bianchi, M. L., Trugilho, P. F., Höfler, J., \& Pereira, A. J. (2015). Análise das propriedades da madeira e do carvão vegetal de híbridos de eucalipto em três idades. Cerne, 21(2), 191-197. http://dx.doi.org/10.1590/01047760201521021294.

Souza, P. F., Silva, J. A., Lucena, D. D. S., Santos, W. D. S., Henriques, Í. G. N., Lucena, M. F. D. A., \& Souza, A. D. (2016). Estudos fitossociológicos e dendrométricos em um fragmento de Caatinga, São José de Espinharas - PB. Ciência Florestal, 26(4), 1317-1330. http://dx.doi.org/10.5902/1980509825152.

Tavares, S. R. L. (Ed.). (2014). Biocombustíveis sólidos: fonte energética alternativa visando à recuperação de áreas degradadas e à conservação do Bioma Caatinga. Brasília: Editora do IFRN.

Trugilho, P. F., Goulart, S. L., Assis, C. O., Couto, F. B. S., Alves, I. C. N., Protásio, T. P., \& Napoli, A. (2015). Características de crescimento, composição química, física e estimativa de massa seca de madeira em clones e espécies de Eucalyptus jovens. Ciência Rural, 45(4), 661-666. http://dx.doi.org/10.1590/0103-8478cr20130625.

Zakia, M. J. B., Pareyn, F. G., \& Riegelhaupt, E. (1992). Equações de peso e volume para oito espécies lenhosas nativas do Seridó RN (Vol. 1). Natal: PNUD/FAO/IBAMA.

Contribuição dos autores: ACC: conceituação, curadoria de dados, análise formal, obtenção de financiamento, investigação, metodologia, administração do projeto, recursos, software, supervisão, validação, visualização, escrita - primeira redação, escrita - revisão e edição; RCS: obtenção de financiamento, investigação, administração do projeto, recursos, supervisão, validação, escrita - revisão e edição; RVOC: curadoria de dados, análise forma, software, supervisão, validação, visualização, escrita - revisão e edição; CPSS: conceituação, metodologia, visualização, escrita - primeira redação, escrita - revisão e edição; SELC: conceituação, metodologia, visualização, escrita - primeira redação, escrita - revisão e edição; AJEC: conceituação, curadoria de dados, metodologia, recursos; FGCP: conceituação, investigação, metodologia, recursos, supervisão, validação, visualização; GBV: conceituação, análise formal, supervisão, validação, visualização, escrita - revisão e edição; AFDJ: curadoria de dados, análise formal, metodologia, software, supervisão, validação, visualização, escrita - revisão e edição; MNFA: conceituação, metodologia, escrita - primeira redação. 